\title{
Effects of Surface Modification of Nanodiamond Particles for Nucleation Enhancement during Its Film Growth by Microwave Plasma Jet Chemical Vapour Deposition Technique
}

\author{
Chii-Ruey Lin, $, 2,3$ Da-Hua Wei, ${ }^{1,2,3}$ Minh-Khoa BenDao, ${ }^{2,3}$ Hong-Ming Chang, ${ }^{2,3}$ \\ Wei-En Chen, ${ }^{2,3}$ and Jen-Ai Lee ${ }^{4}$ \\ ${ }^{1}$ Department of Mechanical Engineering and Institute of Manufacturing Technology, National Taipei University of Technology, \\ Taipei 106, Taiwan \\ ${ }^{2}$ Graduate Institute of Mechanical and Electrical Engineering, National Taipei University of Technology, Taipei 106, Taiwan \\ ${ }^{3}$ Institute of Mechatronic Engineering, National Taipei University of Technology, Taipei 106, Taiwan \\ ${ }^{4}$ School of Pharmacy, Taipei Medical University, Taipei 11031, Taiwan
}

Correspondence should be addressed to Chii-Ruey Lin; crlin@ntut.edu.tw and Jen-Ai Lee; jenai@tmu.edu.tw

Received 13 September 2013; Revised 12 January 2014; Accepted 13 January 2014; Published 5 May 2014

Academic Editor: Ching-Song Jwo

Copyright (C) 2014 Chii-Ruey Lin et al. This is an open access article distributed under the Creative Commons Attribution License, which permits unrestricted use, distribution, and reproduction in any medium, provided the original work is properly cited.

\begin{abstract}
The seedings of the substrate with a suspension of nanodiamond particles (NDPs) were widely used as nucleation seeds to enhance the growth of nanostructured diamond films. The formation of agglomerates in the suspension of NDPs, however, may have adverse impact on the initial growth period. Therefore, this paper was aimed at the surface modification of the NDPs to enhance the diamond nucleation for the growth of nanocrystalline diamond films which could be used in photovoltaic applications. Hydrogen plasma, thermal, and surfactant treatment techniques were employed to improve the dispersion characteristics of detonation nanodiamond particles in aqueous media. The seeding of silicon substrate was then carried out with an optimized spin-coating method. The results of both Fourier transform infrared spectroscopy and dynamic light scattering measurements demonstrated that plasma treated diamond nanoparticles possessed polar surface functional groups and attained high dispersion in methanol. The nanocrystalline diamond films deposited by microwave plasma jet chemical vapour deposition exhibited extremely fine grain and high smooth surfaces $(\sim 6.4 \mathrm{~nm} \mathrm{rms})$ on the whole film. These results indeed open up a prospect of nanocrystalline diamond films in solar cell applications.
\end{abstract}

\section{Introduction}

Since chemical vapour deposition (CVD) diamond films possess many outstanding properties of diamond with low dimensional structure, they have increasingly attracted interests in the area of nanomanufacturing including semiconductor, optics, biomedical, and biosensor engineering [1-6]. Also, due to other important features including transparency over a wide spectral range, good thermal stability, and wear resistance, the films have been considered as promising candidates to replace silicon and metal in high performance devices which operate in harsh environments. High quality undoped CVD diamond films with $\mathrm{n}$ - and p-type characteristics have been successfully manufactured by the most common techniques including microwave plasma-enhanced chemical vapour deposition (MPECVD) and hot filament chemical vapour deposition (HFCVD) [7-10].

The CVD-deposited diamond film normally consists of diamond grains and grain boundaries containing impurities as well as nondiamond carbon phase. The diamond grains and grain boundaries have significant effects on not only the electrical properties, but also the surface roughness of the diamond films. Obviously, high surface roughness may hinder the performance of the diamond-based devices such as MEMS, biosensor, and p-n junction-based device. In a typical diamond film grown by CVD techniques, the surface roughness is directly related to the diamond nucleation enhancement which is also known as "pretreatment" 
procedure. Till now, the enhancement of the diamond nucleation density for nondiamond substrate can be carried out by various methods including spin-coating with a diamond slurry, plasma precarburization, scratching with $\mathrm{Al}_{2} \mathrm{O}_{3}$ or diamond powders, ultrasonicating with mixture of nanometal and nanodiamond particles (NDPs), and biasenhance nucleation. Among these, the method of spincoating with diamond slurry in air at room temperature is inexpensive, environment friendly, and relatively simple; thus it is appropriate for the growth of large-area diamond film, reduces the production cost, and is suitable for the commercial applications. It should be noted here that the size of particle used for spin-coating procedure also influences roughness of the diamond films, resulting in demand of a highly dispersed aqueous suspension of diamond particles with uniform and ultrafine particle size.

Commercial detonation NDPs with round shape are usually used for nucleation enhancement step in CVD diamond growth [11]. It is well known that the significant impurities from nondiamond carbon and metal existing in postsynthesized carbon soot include NDPs and, however, lead to serious aggregation phenomena of typical ND particles. In this study, we employed various surface modification techniques to improve the dispersion characteristics of NDPs in methanol solution, aiming at the diamond nucleation enhancement step in a typical CVD diamond growth process.

\section{Experimental}

Detonation diamond nanoparticles having 4-6 $\mathrm{nm}$ in mean diameter were purchased from Fact Diamond Company, a famous diamond manufacturing company. The NDPs were first purified by an acid treatment procedure using an acid mixture of $\mathrm{HNO}_{3}: \mathrm{H}_{2} \mathrm{SO}_{4}: \mathrm{H}_{2} \mathrm{O}_{2}=1: 1: 3$ in glass autoclave at $100^{\circ} \mathrm{C}$ for $1 \mathrm{~h}$ with ultrasonicating enhancement. The purified powders were then rinsed in double-distilled water for several times until the disappearance of the dark-yellowish color of the suspension, followed by recovery procedures using centrifuged classification equipment and evaporation in room temperature. The acid-treated diamond nanoparticles are denoted as A-NDPs. The hydrogenation of A-NDPs was carried out using MPECVD system with hydrogen plasma excited at $600 \mathrm{~W}$ and 60 Torr of microwave power and working pressure, respectively. Thermal treatment process was conducted on the A-NDPs at $550^{\circ} \mathrm{C}$ under vacuum ambient for $3 \mathrm{~h}$. Also, in other experiments of A-NDPs with surfactant, $50 \mathrm{mg}$ of diamond particles was dispersed in $40 \mathrm{~mL}\left(\mathrm{CH}_{2}\right)_{4} \mathrm{O}$ (tetrahydrofuran (THF)) as dispersion media added by $30 \mathrm{wt} \%$ oleylamine (OLA). In all examinations of the dispersion of the diamond nanoparticles, the diamond suspension was obtained using $0.75 \mathrm{wt} \%$ NDPs in methanol. The as-received suspensions were then employed for the diamond nucleation enhancement using spin-coating technique. In this study, diamond films were deposited onto nucleated silicon substrate having size of $20 \times 20 \times$ $1 \mathrm{~mm}^{3}$ by microwave plasma jet chemical vapour deposition (MPJCVD) system at 35 Torr and with gas mixture of $8 \%$ $\mathrm{CH}_{4}: 92 \% \mathrm{H}_{2}$ as precursors. The deposition time was $2 \mathrm{~h}$.

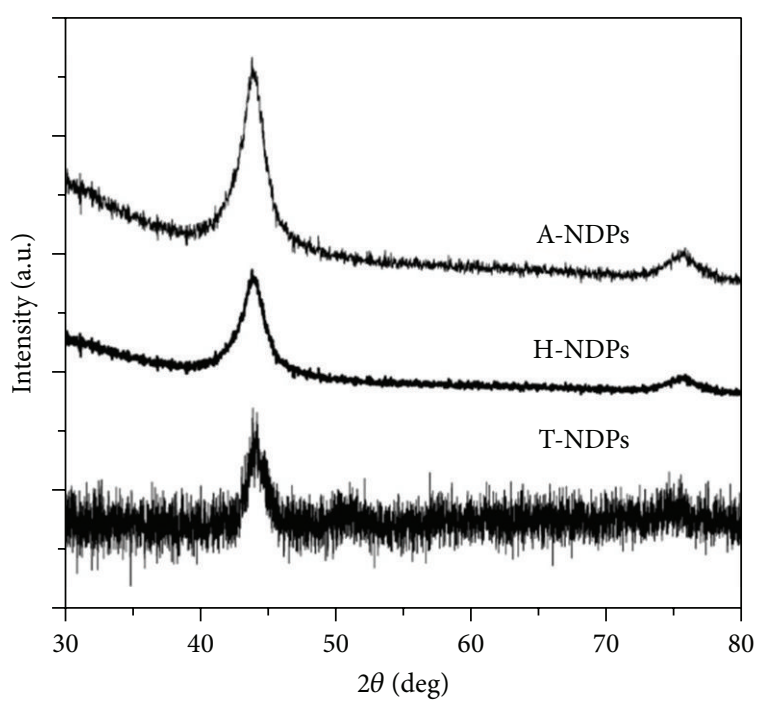

FIGURE 1: X-ray diffraction patterns for the diamond nanoparticles after different surface modification procedures using harsh acid (A-NDPs), hydrogen plasma (H-NDPs), and thermal (T-NDPs) treatments.

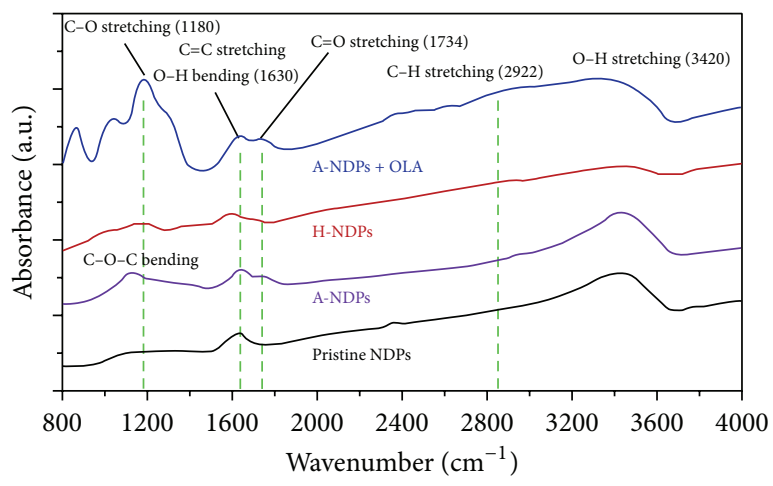

FIGURE 2: FT-IR spectra of pristine NDPs and the diamond particles treated by harsh acid (A-NDPs), hydrogen plasma (H-NDPs), and oleylamine solvent.

X-ray diffractometer (XRD) with $\mathrm{CuK}_{\alpha}$ radiation $(\lambda=$ $0.1542 \mathrm{~nm}$ ) was employed to characterize the effect of the hydrogen plasma and thermal treatment procedures on the crystallinity of the NDPs. Dynamic light scattering (DLS) was used to investigate the dispersion of the NDPs in organic solution. Fourier transform infrared (FT-IR) was conducted with $\mathrm{KBr}$ method to analyze the surface functional groups of the as-prepared NDPs. Scanning electron microscope (SEM) and transmission electron microscope (TEM, JEOL JEM-2100F) were used to examine the characteristics of the nanodiamond particles.

\section{Results and Discussion}

The effects of the harsh acid treatment and surface modification procedures on the structural alteration of NDPs were investigated by X-ray diffraction. Figure 1 shows the XRD patterns of the diamond particle samples treated with 


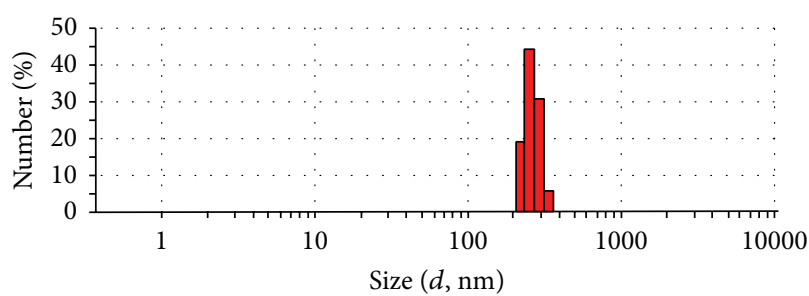

(a)

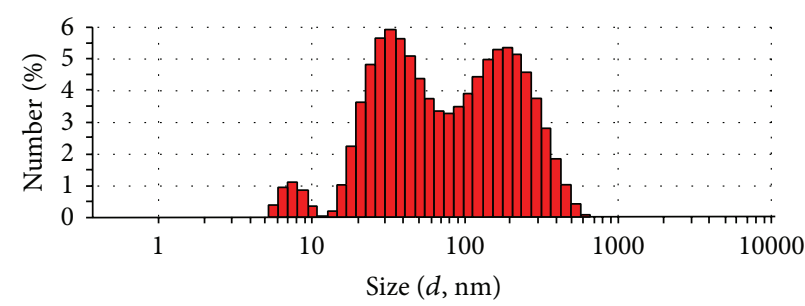

(c)

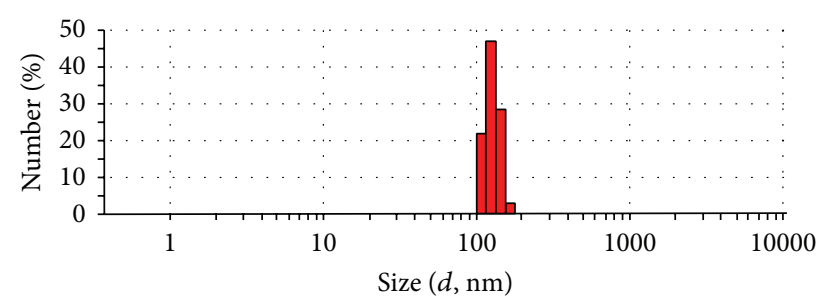

(b)

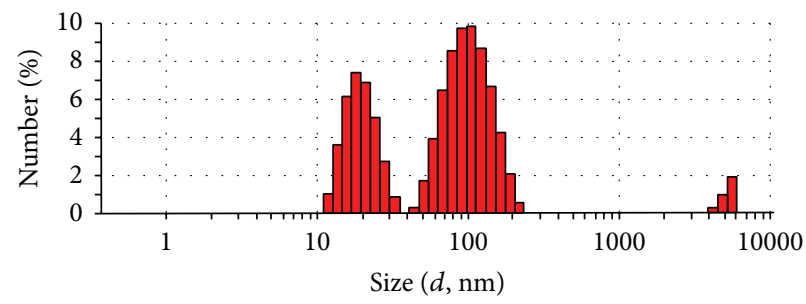

(d)

FIGURE 3: Size distribution of NDPs after treatment procedures using (a) harsh acid mixture, (b) vacuum annealing, (c) OLA surfactant, and (d) hydrogen plasma.

harsh acid (A-NDPs), hydrogen plasma (H-NDPs), and postannealing procedure (T-NDPs). All spectra show the characteristic peak of the (111) crystalline orientation of the diamond structure ( $2 \theta=43.935^{\circ}$, JCPDS number 89-3441). The diffraction peak of the acid-treated NDPs with high intensity exhibits highly crystalline structure of the diamond particles. The XRD pattern of the A-NDPs also demonstrated that the heavy metal and nondiamond carbon impurities that existed in pristine diamond particle samples, on the whole, were thoroughly removed by the acid treatment procedures. After $1 \mathrm{~h}$ of hydrogenation process, the diffraction intensity of diamond peak turned to be weaker and broader due to the graphitization induced by microwave plasma; however, a further analysis to confirm this suggestion is needed. In both A-NDPs and H-NDPs, the crystallite size of the diamond particles was estimated through the full width at half maximum (FWHM) and in qualitative agreement with the characteristics of the commercial diamond nanoparticles synthesized by detonation method which have size range of 5-10 nm. In other words, the hydrogenation used had no remarkable effects on the size of the diamond core structure of the NDPs. The diffraction line of the sample annealed in vacuum ambient for $3 \mathrm{~h}$ at $550^{\circ} \mathrm{C}$ was relatively weak with higher intensity of the noise signals revealing the formed noncubic diamond and impurities component, exhibiting the surface graphitization as well as a harsh oxidization of diamond particles.

Figure 2 shows the FT-IR spectra of the pristine diamond particles and the obtained samples after acid treatment, hydrogenation, and surface modification using OLA solvent as surfactant. The spectra of pristine NDPs exhibited the main features related to $\mathrm{C}=\mathrm{C}\left(1625 \mathrm{~cm}^{-1}\right)$ and $\mathrm{O}-\mathrm{H}$ stretching vibration $\left(3450 \mathrm{~cm}^{-1}\right)$. The apparent peak at $1734 \mathrm{~cm}^{-1}$ in the spectra of the samples treated with harsh acid mixture indicates the induced $\mathrm{C}=\mathrm{O}$ stretching as result of the conversions of original functional groups such as alcohols into carboxylic acids. Also, the apparent $\mathrm{C}-\mathrm{O}-\mathrm{C}$ bending $\left(1105 \mathrm{~cm}^{-1}\right)$ reveals the formation of the carbonyl groups on the surface of the NDPs which may have resulted from the ether bonds between NDPs during the acid treatment procedure. This also can be observed in other oxidation processes. The FT-IR spectra of the hydrogenated NDPs show the significant blue shift and weakening of $\mathrm{C}=\mathrm{C}$ stretching, indicating the hydrogen etching of graphite phase and nondiamond impurities ingredient in the NDPs samples. These results demonstrate that the hydrogenation using microwave plasma contributed to the elimination of hydrophobic surface functional groups such carbonyl and carboxyl. For the A-NDPs modified with OLA solvent, the spectra show the added groups on the surface of diamond particle corresponding to $\mathrm{C}-\mathrm{O}$ stretching $\left(1180 \mathrm{~cm}^{-1}\right)$ and the deformation $\mathrm{O}-\mathrm{H}$ bending $\left(1630 \mathrm{~cm}^{-1}\right)$ which were assigned to the esters, alcohols, and aldehydes.

Figure 3 shows the dispersion characteristics of NDPs samples obtained after different surface modification in aqueous solution at $\mathrm{pH}$ 7. The acid-treated NDPs exhibit severe agglomeration with about $300 \mathrm{~nm}$ in dimension. After the annealing procedure in vacuum ambient at $550^{\circ} \mathrm{C}$, the agglomeration size becomes smaller than $200 \mathrm{~nm}$ as over $45 \%$ of the particles have measured size range of $140 \mathrm{~nm}$. The NDPs treated with OLA surfactant and hydrogen plasma possess higher dispersion in methanol solution which have wide particle size distribution and are consistent with the aforementioned surface characterization. The existent agglomeration of NDPs in solution may be led by the C-O-C bonding between adjacent particles which was observed in the FT-IR spectra.

Figures 4(a) and 4(b) show the TEM image of the NDPs treated with OLA surfactant and hydrogen plasma, which demonstrates that the prepared samples have highly dispersive property in the methanol solution. Moreover, the hydrogenated nanoparticles seem to have better dispersion while agglomeration can be observed in the OLA treated 

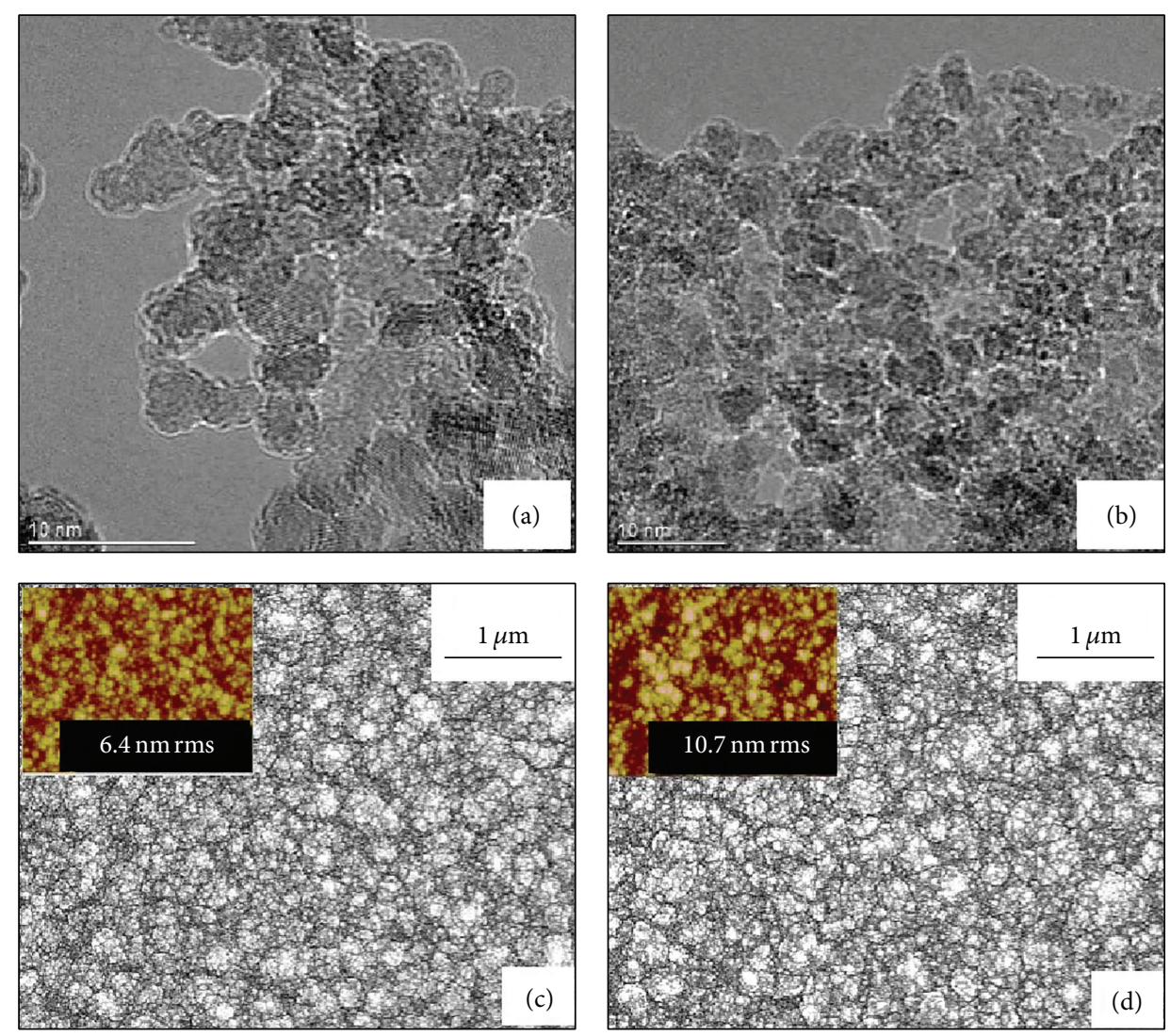

FIGURE 4: High resolution TEM image of NDPs treated by (a) hydrogen plasma and (b) OLA surfactant and the nanocrystalline diamond films grown with nucleation enhancement using suspension of (c) H-NDPs and (d) OLA treated NDPs.

diamond sample. The diamond nanoparticles suspension was further employed in diamond nucleation enhancement step for nanocrystalline diamond films growth using spin-coating technique. The morphology of the as-prepared diamond films is shown in plan-view SEM. As shown in Figure 4(c), the diamond films growth with the nucleation enhancement by H-NDPs suspension exhibits a smooth morphology with very fine grains less than $40 \mathrm{~nm}$ in size and without any grain cluster. The films growth from the nucleated substrate using OLA treated suspension possesses coarser diamond grain and higher surface roughness $(10.7 \mathrm{~nm})$, as shown in Figure 4(d). These above results confirm the aforementioned trends on the size distribution of the NDPs in methanol.

\section{Conclusion}

In this study, various surface modification methods for commercial nanodiamond particles have been employed to improve their dispersion in aqueous solution, aiming at high performance diamond nucleation enhancement for nanocrystalline diamond films growth with low surface roughness. The hydrogen plasma was found to significantly improve the dispersive of the NDPs due to the elimination of the contained covalent bonds and hydrophobic functional groups, while OLA could be good surfactant for diamond suspension due to the added hydrophilic surface functional groups. The hydrogenation also had no remarkable effects on the crystalline structure of the diamond particles but contributed to the etching of the existed nondiamond phases. The deposited diamond films with nucleation enhancement using the as-prepared diamond suspension exhibited highly smooth surface whose measured surface roughness was $7 \mathrm{~nm}$ below. The achieved results demonstrate an avenue to scale up the production of diamond films for solar cell applications.

\section{Conflict of Interests}

The authors declare that there is no conflict of interests regarding the publication of this paper.

\section{Acknowledgments}

This work was financially supported by the Main Research Project of the National Science Council of China under Grant no. NSC 102-2221-E-027-040-MY2 and the Cooperation Project of National Taipei University of Technology and Taipei Medical University under Grant no. NTUT-TMU-101-07.

\section{References}

[1] D. R. Mumm, K. T. Faber, M. D. Drory, and C. F. Gardinier, "High-temperature hardness of chemically vapor-deposited 
diamond," Journal of the American Ceramic Society, vol. 76, no. 1, pp. 238-240, 1993.

[2] M. Akaishi, S. Yamaoka, J. Tanaka, T. Ohsawa, and O. Fukunaga, "Synthesis of sintered diamond with a high electrical resitivity and high hardness," Materials Science and Engineering, vol. 105106, no. 2, pp. 517-523, 1988.

[3] D. M. Bhusari, J. R. Yang, T. Y. Wang, K. H. Chen, S. T. Lin, and L. C. Chen, "Effects of substrate pretreatment and methane fraction on the optical transparency of nanocrystalline diamond thin films," Journal of Materials Research, vol. 13, no. 7, pp. 1769-1773, 1998.

[4] H. Bai, N. Ma, J. Lang, C. Zhu, and Y. Ma, “Thermal conductivity of $\mathrm{Cu}$ /diamond composites prepared by a new pretreatment of diamond powder," Composites Part B: Engineering, vol. 52, pp. 182-186, 2013.

[5] C.-R. Lin, W.-H. Liao, D.-H. Wei, J.-S. Tsai, C.-K. Chang, and W.-C. Fang, "Formation of ultrananocrystalline diamond films with nitrogen addition," Diamond and Related Materials, vol. 20, no. 3, pp. 380-384, 2011.

[6] W. H. Liao, C. R. Lin, D. H. Wei et al., "Concurrent improvement in biocompatibility and bioinertness of diamond-like carbon films with nitrogen doping," Journal of Biomedical Materials Research A, vol. 100, no. 11, pp. 3151-3156, 2012.

[7] C. R. Lin, D. H. Wei, M. K. BenDao, R. J. Chung, and H. M. Chang, "Nanocrystalline diamond particles prepared by highenergy ball milling method," Applied Mechanics and Materials, vol. 284-287, pp. 168-172, 2013.

[8] Y. H. Dong, R. Q. Zhang, and Z. G. Ye, "Effect of diamond on the thermal conductivities of diamond-copper composites prepared by spark plasma sintering," Advanced Materials Research, vol. 532-533, pp. 25-29, 2012.

[9] H. Gomez, C. L. Frewin, A. Kumar, S. E. Saddow, and C. Locke, "Study of the adhesion and biocompatibility of nanocrystalline diamond (NCD) films on 3C-SiC substrates," Materials Research Society Symposium Proceedings, vol. 1203, pp. 179-184, 2010.

[10] M. Amaral, P. S. Gomes, M. A. Lopes, J. D. Santos, R. F. Silva, and M. H. Fernandes, "Nanocrystalline diamond as a coating for joint implants: cytotoxicity and biocompatibility assessment," Journal of Nanomaterials, vol. 2008, Article ID 894352, 9 pages, 2008.

[11] W. H. Liao, C. R. Lin, and D. H. Wei, "Effect of $\mathrm{CH}_{4}$ concentration on the growth behavior, structure, and transparent properties of ultrananocrystalline diamond films synthesized by focused microwave $\mathrm{Ar} / \mathrm{CH}_{4} / \mathrm{H}_{2}$ plasma jets," Applied Surface Science, vol. 270, pp. 324-330, 2013. 

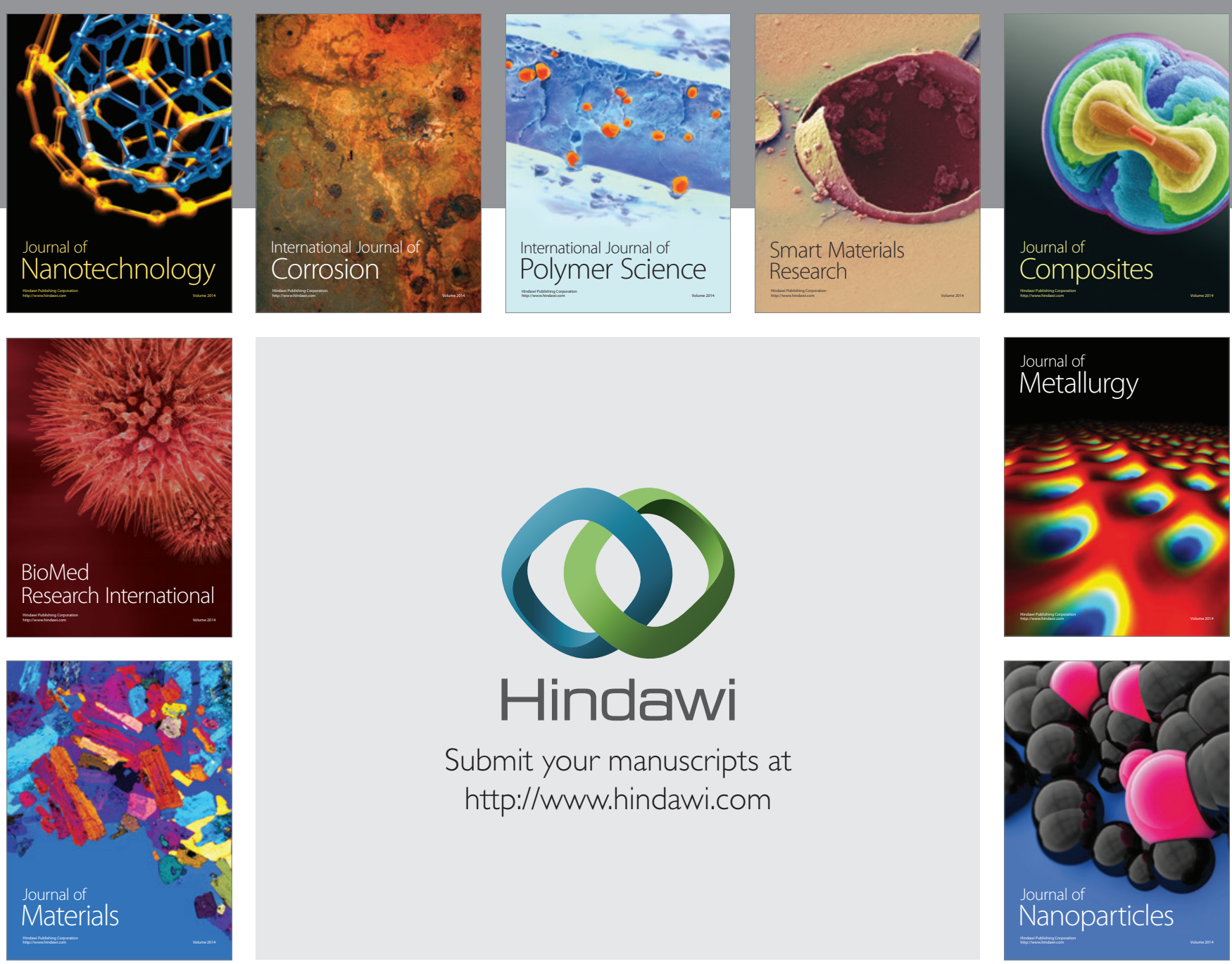

Submit your manuscripts at http://www.hindawi.com
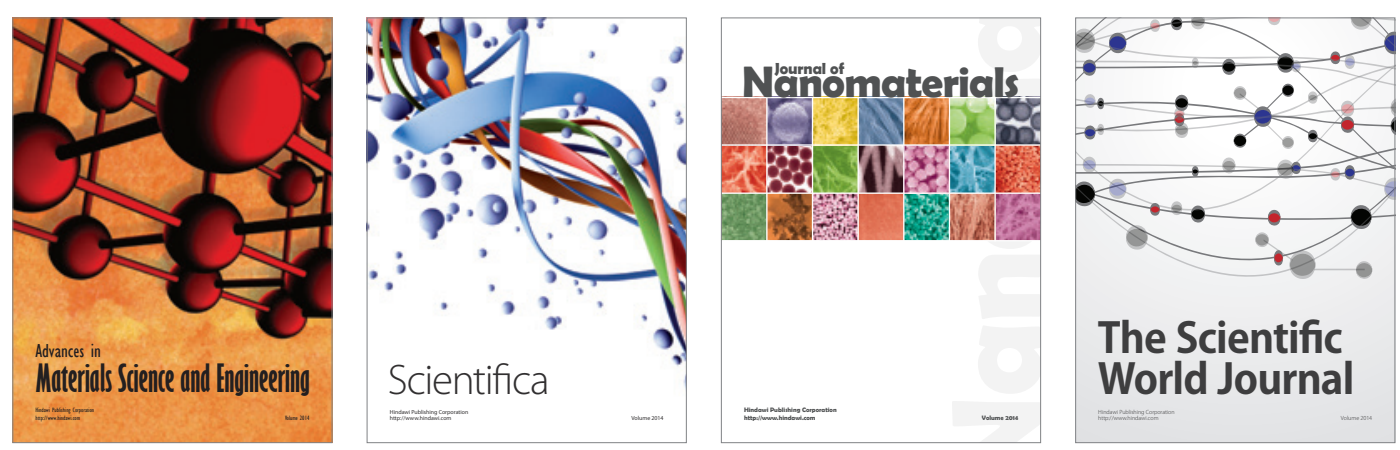

\section{The Scientific World Journal}
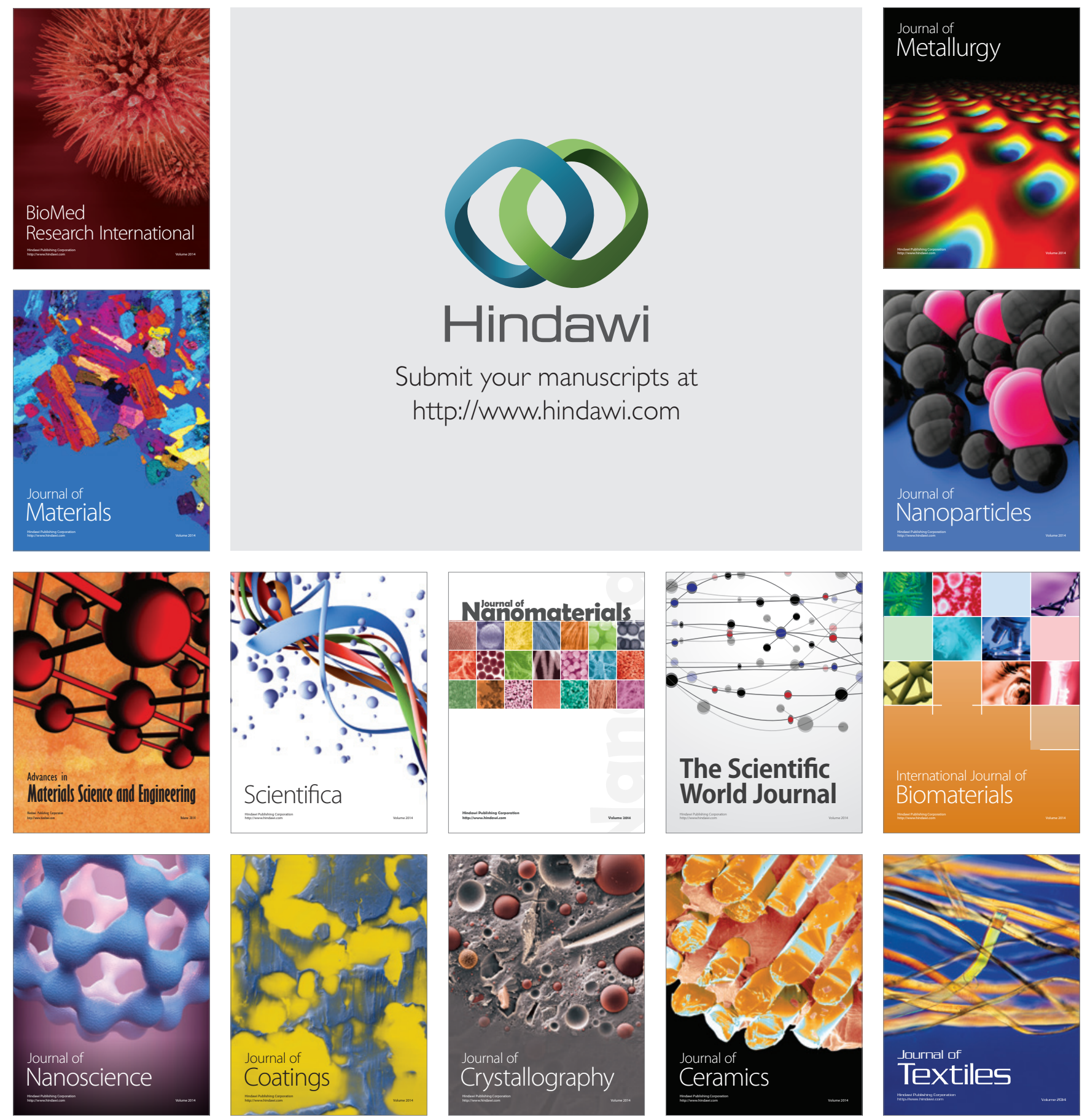